\title{
The Scattering of Electromagnetic Waves from Two-Dimensional Randomly Rough Perfectly Conducting Surfaces: The Full Angular Intensity Distribution
}

\author{
Ingve Simonser* \\ Department of Physics, Norwegian University of Science and Technology (NTNU), NO-7491 Trondheim, Norway \\ Alexei A. Maradudin $\dagger^{\dagger}$ and Tamara A. Leskova \\ Department of Physics and Astronomy and Institute for Surface and Interface Science, \\ University of California, Irvine CA 92697, U.S.A.
}

(Dated: October 29, 2018)

\begin{abstract}
By a computer simulation approach we study the scattering of $p$ - or $s$-polarized light from a twodimensional, randomly rough, perfectly conducting surface. The pair of coupled inhomogeneous integral equations for two independent tangential components of the magnetic field on the surface are converted into matrix equations by the method of moments, which are then solved by the biconjugate gradient stabilized method. The solutions are used to calculate the mean differential reflection coefficient for given angles of incidence and specified polarizations of the incident and scattered fields. The full angular distribution of the intensity of the scattered light is obtained for strongly randomly rough surfaces by a rigorous computer simulation approach.
\end{abstract}

PACS numbers: 42.25.-p; 41.20.-q

\section{INTRODUCTION}

Theoretical/computational studies of the scattering of light from two-dimensional randomly rough perfectly conducting surfaces are carried out primarily for two reasons. These are that a perfectly conducting surface is a good approximation to a finitely conducting surface in the far infrared region of the optical spectrum, but computationally less intensive to study than a finitely conducting surface, and that the development of computational methods for calculations of scattering from rough perfectly conducting surfaces can serve as the first step in the development of methods that can be used in calculations of scattering from rough finitely conducting surfaces.

In the earliest numerical studies of the scattering of light from a two-dimensional randomly rough perfectly conducting surface [1], the pair of coupled inhomogeneous integral equations for two independent tangential components of the total magnetic field on the rough surface obtained from scattering theory was first converted into a pair of coupled inhomogeneous matrix equations by the methods of moments [2]. The system of matrix equations was then solved by Neumann-Liouville iteration. This is a formally exact approach, but one that is computationally intensive. It is an $O\left(M N^{2}\right)$ approach, where $N$ is the number of unknowns to be determined and $M$ is the number of iterations

Subsequent work on this problem has proceeded in two directions. One is the exact solution of the integral equa-

\footnotetext{
*Electronic address: Ingve.Simonsen@phys.ntnu.no URL: http: //web.phys.ntnu.no/ ingves

'Electronic address: aamaradu@uci.edu

${ }^{\ddagger}$ Electronic address: tleskova $@$ uci.edu
}

tions of scattering theory by numerical methods that are faster than a straightforward application of the method of moments followed by an iterative solution of the resulting matrix equation. For example, Wagner et al. 3] have developed a fast multipole Fast Fourier Transform method to calculate the scattering of an electromagnetic wave from a small height two-dimensional randomly rough perfectly conducting surface that is an $O(N \ln N)$ method. For rougher two-dimensional perfectly conducting surfaces they have shown that the multi-level fast multipole algorithm, also an $O(N \ln N)$ method, is more efficient.

The other direction that has been taken is the approximate solution of the exact integral equations. In the sparse-matrix flat-surface iterative approach of Tsang et al. 4, 5, the matrix elements connecting two close points on the surface are treated exactly, while those connecting two distant points are treated approximately, in an iterative solution of the matrix equations obtained by the method of moments. This approach has been applied to the study of the scattering of electromagnetic waves from a two-dimensional randomly rough perfectly conducting surface [6, 7]. It has been elaborated and made faster by Johnson and his colleagues, resulting in an $O(N)$ method in some cases, and has been applied to the scattering of electromagnetic waves from a two-dimensional randomly rough perfectly conducting surface [8]. Soriano and Saillard [9] have developed a sparse-matrix flat-surface iterative approach, in which the matrix equations are solved by an iterative Krylov method, the biconjugate gradient stabilized method 10 .

In this paper we return to the approach used in [1, where the sparse-matrix flat-surface approximation is not used: the matrix elements connecting two points are calculated accurately for all separations of the two points. However, the resulting matrix equations are solved here by the biconjugate gradient stabilized method instead of by Neumann-Liouville iteration, as in [1]. We show that 
this approach, together with the increase in computational power since [1] was written, provides a simple and reliable way of calculating the mean differential reflection coefficient for given angles of incidence and specified polarizations of the incident and scattered fields, with a modest expenditure of CPU time.

This paper is organized as follows: We start by presenting the scattering geometry considered (Sec. II) followed by the mathematical formulation of the scattering problem (Sec. III), including the central integral equation on which the computer simulations are based. Section IV] is devoted to the presentation and discussion of the numerical results obtained from a rigorous computer simulation approach based on an integral equation for the surface currents derived in Sec. III. A detailed discussion of the numerical aspects of such calculations is given in Sec. V Finally the conclusions that can be drawn from this work are presented in Sec. VI.

\section{SCATTERING GEOMETRY}

The physical system we consider in this work consists of vacuum in the region $x_{3}>\zeta\left(\mathbf{x}_{\|}\right)$, where $\mathbf{x}_{\|}=$ $\left(x_{1}, x_{2}, 0\right)$, and a perfect conductor in the region $x_{3}<$ $\zeta\left(\mathbf{x}_{\|}\right)$[Fig. 1]. The surface profile function $\zeta\left(\mathbf{x}_{\|}\right)$is assumed to be a single-valued function of $\mathbf{x}_{\|}$that is differentiable with respect to $x_{1}$ and $x_{2}$, and constitutes a stationary, zero-mean, isotropic, Gaussian random process defined by $\left\langle\zeta\left(\mathbf{x}_{\|}\right) \zeta\left(\mathbf{x}_{\|}^{\prime}\right)\right\rangle=\delta^{2} W\left(\left|\mathbf{x}_{\|}-\mathbf{x}_{\|}^{\prime}\right|\right)$, where the angle brackets denote an average over the ensemble of realizations of the surface profile function, and $\delta=\left\langle\zeta^{2}\left(\mathbf{x}_{\|}\right)\right\rangle^{\frac{1}{2}}$ is the rms height of the surface. In the numerical calculations carried out in the present work we will assume a Gaussian form for $W\left(\left|\mathbf{x}_{\|}-\mathbf{x}_{\|}^{\prime}\right|\right)$, namely $W\left(\left|\mathbf{x}_{\|}-\mathbf{x}_{\|}^{\prime}\right|\right)=\exp \left[-\left(\mathbf{x}_{\|}-\mathbf{x}_{\|}^{\prime}\right)^{2} / a^{2}\right]$, where $a$ is the transverse correlation length of the surface roughness. Each realization of the surface profile function with these properties is generated numerically by a two-dimensional version of the filtering method used in 11.

\section{FORMULATION}

\section{A. Integral Equation}

The starting point for our analysis is the Stratton-Chu formula [12] for the magnetic field in the vacuum,

$$
\begin{aligned}
\theta\left(x_{3}\right. & \left.-\zeta\left(\mathbf{x}_{\|}\right)\right) \mathbf{H}^{>}(\mathbf{x} \mid \omega)=\mathbf{H}(\mathbf{x} \mid \omega)_{i n c} \\
& +\frac{1}{4 \pi} \int d^{2} x_{\|}^{\prime}\left[\nabla^{\prime} g_{0}\left(\mathbf{x} \mid \mathbf{x}^{\prime}\right)\right]_{x_{3}^{\prime}=\zeta\left(\mathbf{x}_{\|}^{\prime}\right)} \times \mathbf{J}_{H}\left(\mathbf{x}_{\|}^{\prime} \mid \omega\right),
\end{aligned}
$$

where $\theta(z)$ is the Heaviside unit step function, and $\mathbf{H}(\mathbf{x} \mid \omega)_{\text {inc }}$ is the magnetic component of the incident field.

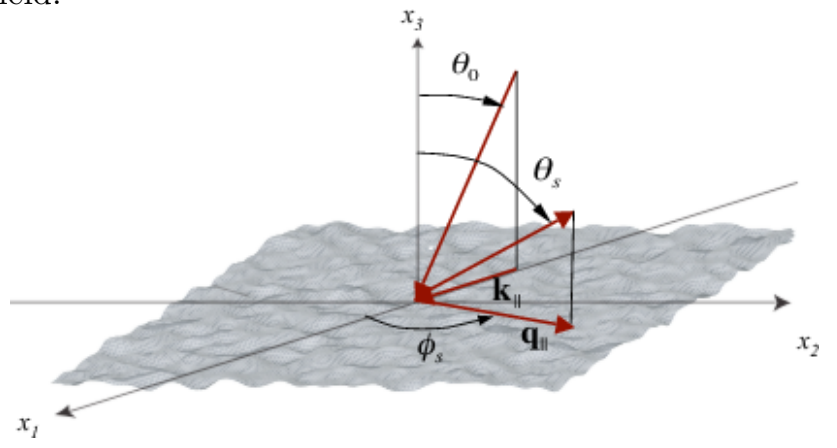

FIG. 1: (Color online) A sketch of the scattering geometry considered in the present work, where the coordinate system used and angles of incidence and scattering are defined.

The function $g_{0}\left(\mathbf{x} \mid \mathbf{x}^{\prime}\right)$ is the scalar free-space Green's function and has the representations

$$
\begin{aligned}
g_{0}\left(\mathbf{x} \mid \mathbf{x}^{\prime}\right)= & \frac{\exp \left[i \frac{\omega}{c}\left|\mathbf{x}-\mathbf{x}^{\prime}\right|\right]}{\left|\mathbf{x}-\mathbf{x}^{\prime}\right|} \\
= & \int \frac{d^{2} q_{\|}}{(2 \pi)^{2}} \frac{2 \pi i}{\alpha_{0}\left(q_{\|}\right)} \exp \left[i \mathbf{q}_{\|} \cdot\left(\mathbf{x}_{\|}-\mathbf{x}_{\|}^{\prime}\right)\right] \\
& \times \exp \left[i \alpha_{0}\left(q_{\|}\right)\left|x_{3}-x_{3}^{\prime}\right|\right],
\end{aligned}
$$

where

$\alpha_{0}\left(q_{\|}\right)=\sqrt{\left(\frac{\omega}{c}\right)^{2}-q_{\|}^{2}}, \quad \operatorname{Re} \alpha_{0}\left(q_{\|}\right)>0, \operatorname{Im} \alpha_{0}\left(q_{\|}\right)>0$

and $\omega$ and $c$ are the frequency and speed of light in vacuum. In writing Eq. (1) we have assumed the time dependence $\exp (-i \omega t)$ for the field, but have not indicated this explicitly. The (electric) surface current density $\mathbf{J}_{H}\left(\mathbf{x}_{\|} \mid \omega\right)$ is defined by $\mathbf{J}_{H}\left(\mathbf{x}_{\|} \mid \omega\right)=[\mathbf{n} \times$ $\left.\mathbf{H}^{>}(\mathbf{x} \mid \omega)\right]\left.\right|_{x_{3}=\zeta\left(\mathbf{x}_{\|}\right)}$, where $\mathbf{n}=\left(-\zeta_{1}\left(\mathbf{x}_{\|}\right),-\zeta_{2}\left(\mathbf{x}_{\|}\right), 1\right)$ is a vector that is normal to the surface $x_{3}=\zeta\left(\mathbf{x}_{\|}\right)$at each point of it, directed into the vacuum, and we have introduced the notation $\zeta_{j}\left(\mathbf{x}_{\|}\right)=\partial \zeta\left(\mathbf{x}_{\|}\right) / \partial x_{j}(j=1,2)$.

On evaluating Eq. (1) at $x_{3}=\zeta\left(\mathbf{x}_{\|}\right)+\eta$ and at $x_{3}=\zeta\left(\mathbf{x}_{\|}\right)-\eta$, where $\eta$ is a positive infinitesimal, adding the resulting two equations, and taking the vector cross product of the sum with $\mathbf{n}$, we obtain the integral equation satisfied by the surface current $\mathbf{J}_{H}\left(\mathbf{x}_{\|} \mid \omega\right)$, 


$$
\mathbf{J}_{H}\left(\mathbf{x}_{\|} \mid \omega\right)=2 \mathbf{J}_{H}^{(i)}\left(\mathbf{x}_{\|} \mid \omega\right)+\frac{1}{2 \pi} P \int d^{2} x_{\|}^{\prime} \mathbf{n} \times\left(\llbracket \nabla^{\prime} g_{0}\left(\mathbf{x} \mid \mathbf{x}^{\prime}\right) \rrbracket \times \mathbf{J}_{H}\left(\mathbf{x}_{\|}^{\prime} \mid \omega\right)\right)
$$

where $\mathbf{J}_{H}^{(i)}\left(\mathbf{x}_{\|} \mid \omega\right)=\mathbf{n} \times\left.\mathbf{H}(\mathbf{x} \mid \omega)_{i n c}\right|_{x_{3}=\zeta\left(\mathbf{x}_{\|}\right)}, P$ denotes the Cauchy principal value, and we have simplified the notation by introducing the definition

$$
\llbracket f\left(\mathbf{x} \mid \mathbf{x}^{\prime}\right) \rrbracket=\left.f\left(\mathbf{x} \mid \mathbf{x}^{\prime}\right)\right|_{\substack{x_{3}=\zeta\left(\mathbf{x}_{\|}\right) \\ x_{3}^{\prime}=\zeta\left(\mathbf{x}_{\|}^{\prime}\right)}} .
$$

The system of three equations (4) can be reduced to a system of two equations through the use of the con- dition $\mathbf{n} \cdot \mathbf{J}_{H}\left(\mathbf{x}_{\|} \mid \omega\right)=0$. Thus only two components of $\mathbf{J}_{H}\left(\mathbf{x}_{\|} \mid \omega\right)$ are independent. We choose $\mathbf{J}_{H}\left(\mathbf{x}_{\|} \mid \omega\right)_{1,2}$ as the independent components, while

$$
J_{H}\left(\mathbf{x}_{\|} \mid \omega\right)_{3}=\zeta_{1}\left(\mathbf{x}_{\|}\right) J_{H}\left(\mathbf{x}_{\|} \mid \omega\right)_{1}+\zeta_{2}\left(\mathbf{x}_{\|}\right) J_{H}\left(\mathbf{x}_{\|} \mid \omega\right)_{2} .
$$

From Eq. (4) we find with the aid of Eq. (6) that the components $\mathbf{J}_{H}\left(\mathbf{x}_{\|} \mid \omega\right)_{1,2}$ satisfy the following pair of equations:

$$
\begin{gathered}
J_{H}\left(\mathbf{x}_{\|} \mid \omega\right)_{1}=2 J_{H}^{(i)}\left(\mathbf{x}_{\|} \mid \omega\right)_{1}-\frac{1}{2 \pi} P \int d^{2} x_{\|}^{\prime}\left\{\left[g_{3}^{(0)}\left(\mathbf{x}_{\|} \mid \mathbf{x}_{\|}^{\prime}\right)-g_{1}^{(0)}\left(\mathbf{x}_{\|} \mid \mathbf{x}_{\|}^{\prime}\right) \zeta_{1}\left(\mathbf{x}_{\|}^{\prime}\right)-\zeta_{2}\left(\mathbf{x}_{\|}\right) g_{2}^{(0)}\left(\mathbf{x}_{\|} \mid \mathbf{x}_{\|}^{\prime}\right)\right] J_{H}\left(\mathbf{x}_{\|}^{\prime} \mid \omega\right)_{1}\right. \\
\left.+g_{1}^{(0)}\left(\mathbf{x}_{\|} \mid \mathbf{x}_{\|}^{\prime}\right)\left[\zeta_{2}\left(\mathbf{x}_{\|}\right)-\zeta_{2}\left(\mathbf{x}_{\|}^{\prime}\right)\right] J_{H}\left(\mathbf{x}_{\|}^{\prime} \mid \omega\right)_{2}\right\} \\
J_{H}\left(\mathbf{x}_{\|} \mid \omega\right)_{2}=2 J_{H}^{(i)}\left(\mathbf{x}_{\|} \mid \omega\right)_{2}-\frac{1}{2 \pi} P \int d^{2} x_{\|}^{\prime}\left\{g_{2}^{(0)}\left(\mathbf{x}_{\|} \mid \mathbf{x}_{\|}^{\prime}\right)\left[\zeta_{1}\left(\mathbf{x}_{\|}\right)-\zeta_{1}\left(\mathbf{x}_{\|}^{\prime}\right)\right] J_{H}\left(\mathbf{x}_{\|}^{\prime} \mid \omega\right)_{1}\right. \\
\left.+\left[g_{3}^{(0)}\left(\mathbf{x}_{\|} \mid \mathbf{x}_{\|}^{\prime}\right)-g_{2}^{(0)}\left(\mathbf{x}_{\|} \mid \mathbf{x}_{\|}^{\prime}\right) \zeta_{2}\left(\mathbf{x}_{\|}^{\prime}\right)-\zeta_{1}\left(\mathbf{x}_{\|}\right) g_{1}^{(0)}\left(\mathbf{x}_{\|} \mid \mathbf{x}_{\|}^{\prime}\right)\right] J_{H}\left(\mathbf{x}_{\|}^{\prime} \mid \omega\right)_{2}\right\}
\end{gathered}
$$

where

$$
g_{l}^{(0)}\left(\mathbf{x}_{\|} \mid \mathbf{x}_{\|}^{\prime}\right)=\llbracket \frac{\partial}{\partial x_{l}} g_{0}\left(\mathbf{x} \mid \mathbf{x}^{\prime}\right) \rrbracket=\left.\left(x_{l}-x_{l}^{\prime}\right)\left[\frac{i(\omega / c)}{\left|\mathbf{x}-\mathbf{x}^{\prime}\right|^{2}}-\frac{1}{\left|\mathbf{x}-\mathbf{x}^{\prime}\right|^{3}}\right] \exp \left[i(\omega / c)\left|\mathbf{x}-\mathbf{x}^{\prime}\right|\right]\right|_{\substack{x_{3}=\zeta\left(\mathbf{x}_{\|}\right) \\ x_{3}^{\prime}=\zeta\left(\mathbf{x}_{\|}^{\prime}\right)}} .
$$

Equations (7) are solved by converting them into a pair of coupled matrix equations. This is done by generating a realization of the surface profile function on a grid of $N^{2}$ points within a square region of the $x_{1} x_{2}$ plane of edge $L$, where the ratio $L / N=\Delta x$ is chosen to be $\Delta x=\lambda / 7$, with $\lambda$ the wavelength of the incident field. The integrals over this region in Eqs. (7) are carried out by means of a two-dimensional version of the extended midpoint method [13, and the values of $\mathbf{J}_{H}\left(\mathbf{x}_{\|} \mid \omega\right)_{1,2}$ are calculated at the points of this grid. The resulting matrix equations are then solved by means of the biconjugate gradient stabilized method $\left[10\right.$. Once $\mathbf{J}_{H}\left(\mathbf{x}_{\|} \mid \omega\right)_{1}$ and $\mathbf{J}_{H}\left(\mathbf{x}_{\|} \mid \omega\right)_{2}$ have been obtained in this way, $\mathbf{J}_{H}\left(\mathbf{x}_{\|} \mid \omega\right)_{3}$ is obtained from Eq. (6).

\section{B. Scattered Field}

With the surface current $\mathbf{J}_{H}\left(\mathbf{x}_{\|} \mid \omega\right)$ in hand, one is ready to start approaching the calculation of the scattered field. To this end, let us start by writing the scattered electric field (in the far zone) in the form

$$
\begin{aligned}
\mathbf{E}(\mathbf{x} \mid \omega)_{s c} & =\int \frac{d^{2} q_{\|}}{(2 \pi)^{2}} \mathcal{E}\left(\mathbf{q}_{+}, \omega\right) \exp \left[i \mathbf{q}_{+} \cdot \mathbf{x}\right] \\
& =\int \frac{d^{2} q_{\|}}{(2 \pi)^{2}}\left[\mathcal{E}_{p}\left(\mathbf{q}_{+}, \omega\right) \hat{\gamma}_{p}\left(\mathbf{q}_{+}, \omega\right)+\mathcal{E}_{s}\left(\mathbf{q}_{+}, \omega\right) \hat{\gamma}_{s}\left(\mathbf{q}_{+}, \omega\right)\right] \exp \left[i \mathbf{q}_{+} \cdot \mathbf{x}\right]
\end{aligned}
$$

where $\mathcal{E}_{\nu}=\mathcal{E} \cdot \hat{\gamma}_{\nu}(\nu=p, s)$. In writing Eq. (9) we have introduced the (unit) polarization vectors $\hat{\gamma}_{\nu}\left(\mathbf{q}_{ \pm}, \omega\right)$ 
for $p$ - and $s$-polarized scattered light that are mutually orthogonal and also orthogonal to the wave-vector $\mathbf{q}_{ \pm}$. They can, in accordance with Sipe [15], be defined as

$$
\begin{aligned}
\hat{\gamma}_{s}\left(\mathbf{q}_{ \pm}, \omega\right) & =\frac{\mathbf{q}_{ \pm} \times \hat{\mathbf{x}}_{3}}{\left|\mathbf{q}_{ \pm} \times \hat{\mathbf{x}}_{3}\right|}=\hat{\mathbf{q}}_{\|} \times \hat{\mathbf{x}}_{3} \\
\hat{\gamma}_{p}\left(\mathbf{q}_{ \pm}, \omega\right) & =\hat{\gamma}_{s}\left(\mathbf{q}_{ \pm}, \omega\right) \times \hat{\mathbf{q}}_{ \pm} \\
& =\frac{q_{\|} \hat{\mathbf{x}}_{3} \mp \alpha_{0}\left(q_{\|}, \omega\right) \hat{\mathbf{q}}_{\|}}{\omega / c}
\end{aligned}
$$

where we have introduced the wave-vector for upward $\left(\mathbf{q}_{+}\right)$and downward $\left(\mathbf{q}_{-}\right)$propagating (plane) waves

$$
\mathbf{q}_{ \pm}\left(\mathbf{q}_{\|}, \omega\right)=\mathbf{q}_{\|} \pm \alpha_{0}\left(q_{\|}\right) \hat{\mathbf{x}}_{3}
$$

From Eqs. (10) it is readily shown that the set $\left\{\hat{\gamma}_{p}\left(\mathbf{q}_{ \pm}, \omega\right), \quad \hat{\gamma}_{s}\left(\mathbf{q}_{ \pm}, \omega\right), \quad \hat{\mathbf{q}}_{ \pm}\left(\mathbf{q}_{\|}, \omega\right)\right\} \quad$ forms a (righthanded) orthonormal triad. This implies, for instance, suppressing the function arguments for simplicity, that $\hat{\gamma}_{\mu} \cdot \hat{\gamma}_{\nu}=\delta_{\mu \nu}, \mathbf{q}_{ \pm} \cdot \hat{\gamma}_{\nu}=0$ as well as

$$
\begin{aligned}
\hat{\gamma}_{s} & =\hat{\mathbf{q}}_{ \pm} \times \hat{\gamma}_{p} \\
\hat{\gamma}_{p} & =-\hat{\mathbf{q}}_{ \pm} \times \hat{\gamma}_{s}, \\
\hat{\mathbf{q}}_{ \pm} & =\hat{\gamma}_{p} \times \hat{\gamma}_{s} .
\end{aligned}
$$

With the use of one of the Maxwell's equations (Faraday's law), $\nabla \times \mathbf{E}=i(\omega / c) \mathbf{H}$, and Eqs. (11), it follows from Eq. (9) that the scattered magnetic field can be written

$$
\mathbf{H}(\mathbf{x} \mid \omega)_{s c}=\int \frac{d^{2} q_{\|}}{(2 \pi)^{2}}\left[\mathcal{E}_{p}\left(\mathbf{q}_{+}, \omega\right) \hat{\gamma}_{s}\left(\mathbf{q}_{+}, \omega\right)-\mathcal{E}_{s}\left(\mathbf{q}_{+}, \omega\right) \hat{\boldsymbol{\gamma}}_{p}\left(\mathbf{q}_{+}, \omega\right)\right] \exp \left[i \mathbf{q}_{+} \cdot \mathbf{x}\right]
$$

On the other hand, the scattered magnetic field is also given in terms of the surface current $\mathbf{J}_{H}\left(\mathbf{x}_{\|} \mid \omega\right)$ by the second term on the right-hand side of Eq. (1), and with the use of Eq. (2b) one is led to $(\nu=p, s)$

$$
\mathcal{E}_{\nu}\left(\mathbf{q}_{+}, \omega\right)=-\frac{(\omega / c)}{2 \alpha_{0}\left(q_{\|}\right)} \int d^{2} x_{\|} \hat{\gamma}_{\nu}\left(\mathbf{q}_{+}, \omega\right) \cdot \mathbf{J}_{H}\left(\mathbf{x}_{\|} \mid \omega\right) \exp \left[-i \mathbf{q}_{+} \cdot \mathbf{x}\right] .
$$

The total time-averaged scattered flux is given by the real part of the 3-component of the (complex) Poynting vector $\left(\mathbf{S}^{c}=c /(8 \pi) \mathbf{E} \times \mathbf{H}^{*}\right)$ of the scattered field, integrated over the plane $x_{3}=0$. From the fields in the form of Eqs. (9) and 12 and the use of Eqs. (11) we find that it is given by

$$
P_{s c}=\frac{c^{2}}{8 \pi \omega} \int_{q_{\|}<\frac{\omega}{c}} \frac{d^{2} q_{\|}}{(2 \pi)^{2}} \alpha_{0}\left(q_{\|}\right)\left[\left|\mathcal{E}_{p}\left(\mathbf{q}_{+}, \omega\right)\right|^{2}+\left|\mathcal{E}_{s}\left(\mathbf{q}_{+}, \omega\right)\right|^{2}\right]
$$

and we recall that $\mathbf{q}_{+}=\mathbf{q}_{+}\left(\mathbf{q}_{\|}, \omega\right)$, defined in Eq. $(10 \mathrm{c})$, depends on the parallel momentum $\mathbf{q}_{\|}$. Moreover, the vector $\mathbf{q}_{\|}$is given in terms of the polar and azimuthal scattering angles $\theta_{s}$ and $\phi_{s}$ by

$$
\mathbf{q}_{\|}=\frac{\omega}{c} \sin \theta_{s}\left(\cos \phi_{s}, \sin \phi_{s}, 0\right)
$$

The expression given by Eq. (14) can then be rewritten as

$$
P_{s c}=\frac{c^{2}}{8 \pi \omega} \frac{1}{4 \pi^{2}}\left(\frac{\omega}{c}\right)^{3} \int d \Omega_{s} \cos ^{2} \theta_{s}\left[\left|\mathcal{E}_{p}\left(\mathbf{q}_{+}, \omega\right)\right|^{2}+\left|\mathcal{E}_{s}\left(\mathbf{q}_{+}, \omega\right)\right|^{2}\right],
$$

where $d \Omega_{s}=\sin \theta_{s} d \theta_{s} d \phi_{s}$ is the element of solid angle about the scattering direction $\left(\theta_{s}, \phi_{s}\right)$.

\section{Incident Field}

The incident electric field vector that will be considered in this study, has the form of a (Gaussian) beam propagating in the direction of

$$
\mathbf{k}=\frac{\omega}{c}\left(\sin \theta_{0} \cos \phi_{0}, \sin \theta_{0} \sin \phi_{0},-\cos \theta_{0}\right),
$$

and is represented by a superposition of incoming plane waves

$$
\begin{gathered}
\mathbf{E}(\mathbf{x} \mid \omega)_{i n c}=\int_{q_{\|}<\frac{\omega}{c}} d^{2} q_{\|} \mathcal{E}^{(i)}\left(\mathbf{q}_{-}, \omega\right) \exp \left[i \mathbf{q}_{-} \cdot \mathbf{x}\right] \\
\times W\left(\mathbf{q}_{\|}, \mathbf{k}_{\|}\right)
\end{gathered}
$$


where $W\left(\mathbf{q}_{\|}, \mathbf{k}_{\|}\right)$denotes an envelope (or window) function, here defined as

$$
W\left(\mathbf{q}_{\|}, \mathbf{k}_{\|}\right)=\frac{w^{2}}{2 \pi} \exp \left[-\frac{w^{2}}{2}\left(\mathbf{q}_{\|}-\mathbf{k}_{\|}\right)^{2}\right]
$$

with $w$ its (and the beam's) half width. Note that in the limit of large beam widths $(w \rightarrow \infty)$, the envelope $W\left(\mathbf{q}_{\|}, \mathbf{k}_{\|}\right)$tends towards $\delta\left(\mathbf{q}_{\|}-\mathbf{k}_{\|}\right)$so that, in this limit, the incident beam becomes a plane wave.

A beam as defined by Eqs. (18) does not adhere to the usual definition of $p$ - or $s$-polarized waves since the plane of incidence is not well-defined in this case (except when $w=\infty)$. However, we will still refer to an incident beam of the form given by Eqs. (18) as $p$-polarized if its electric field vector is in the plane "of incidence" defined by the vectors $\mathbf{k}$ and $\hat{\mathbf{x}}_{3}$. Therefore, for a $p$-polarized beam, the projection of its amplitude vector $\mathcal{E}_{p}^{(i)}\left(\mathbf{q}_{-}, \omega\right)$ onto the $x_{1} x_{2}$-plane will be parallel to $\mathbf{k}_{\|}$. Moreover, the vector amplitude for an $s$-polarized beam, $\mathcal{E}_{s}^{(i)}\left(\mathbf{q}_{-}, \omega\right)$, is defined as

$$
\hat{\mathcal{E}}_{s}^{(i)}\left(\mathbf{q}_{-}, \omega\right)=\hat{\mathbf{q}}_{-} \times \hat{\mathcal{E}}_{p}^{(i)}\left(\mathbf{q}_{-}, \omega\right),
$$

similarly to the relation satisfied by the plane-wave polarization vectors $\hat{\gamma}_{\nu}$ (cf. Eq. (11a)).

Since in this work we are concerned exclusively with isotropic surfaces, we will, with no loss of generality, assume that the vector $\mathbf{k}_{\|}$, if non-zero, is parallel to the $x_{1}$ axis, i.e. $\mathbf{k}_{\|}=k_{\|} \hat{\mathbf{x}}_{1}$. Under this assumption the amplitude vector for a $p$-polarized incident beam, $\mathcal{E}_{p}^{(i)}\left(\mathbf{q}_{-}, \omega\right)$, will lie in the $x_{1} x_{3}$-plane, i.e. its second component will be zero, which with the condition $\nabla \cdot \mathbf{E}=0$ (or equivalently $\left.\mathbf{q}_{-} \cdot \mathcal{E}^{(i)}\left(\mathbf{q}_{-}, \omega\right)=0\right)$ leads us to define

$$
\hat{\mathcal{E}}_{p}^{(i)}\left(\mathbf{q}_{-}, \omega\right)=\frac{\alpha_{0}\left(q_{\|}\right) \hat{\mathbf{x}}_{1}+q_{1} \hat{\mathbf{x}}_{3}}{\left[q_{1}^{2}+\alpha_{0}^{2}\left(q_{\|}\right)\right]^{\frac{1}{2}}} .
$$

The amplitude for the corresponding $s$-polarized beam follows from Eq. $(19)$, and, with the use of Eq. $\sqrt{10 \mathrm{c}}$, it can be written as

$$
\hat{\mathcal{E}}_{s}^{(i)}\left(\mathbf{q}_{-}, \omega\right)=\frac{q_{1} q_{2} \hat{\mathbf{x}}_{1}-\left[q_{1}^{2}+\alpha_{0}^{2}\left(q_{\|}\right)\right] \hat{\mathbf{x}}_{2}-q_{2} \alpha_{0}\left(q_{\|}\right) \hat{\mathbf{x}}_{3}}{(\omega / c)\left[q_{1}^{2}+\alpha_{0}^{2}\left(q_{\|}\right)\right]^{\frac{1}{2}}} .
$$

With the beam amplitudes in the form of Eqs. 20 it is readily established that similar relations to those satisfied by the plane-wave polarization vectors (e.g. Eqs. 10 and (11)), also hold for the polarization amplitudes, $\hat{\mathcal{E}}_{\nu}^{(i)}$, of the Gaussian beam.

Moreover, also note that in the limit of a large beam width $(w \rightarrow \infty)$ Eqs. (20) reduce to the plane wave polarization vectors given previously in Eqs. 10 since in this limit $\mathbf{q}_{\|}=\mathbf{k}_{\|}$with $k_{\|}=k_{1}$. This is another reason for associating the vector amplitudes of Eqs. 20) with $p$ and $s$-polarized components, respectively.

With the polarization vectors available for the incident $p$ - and $s$-polarized components of the incident beam, the incident electric field, of given polarization $\nu$, can according to Eqs. (18) and Eqs. 20), be written (assuming unit amplitude for simplicity) in the following form

$$
\begin{gathered}
\mathbf{E}_{\nu}(\mathbf{x} \mid \omega)_{i n c}=\int_{q_{1}<\frac{\omega}{c}} d^{2} q_{\|} \hat{\mathcal{E}}_{\nu}^{(i)}\left(\mathbf{q}_{-}, \omega\right) \exp \left[i \mathbf{q}_{-} \cdot \mathbf{x}\right] \\
\times W\left(\mathbf{q}_{\|}, \mathbf{k}_{\|}\right) .
\end{gathered}
$$

In precisely the same way as Eq. 12 was established for the scattered field, it follows from Eqs. (21) by using Eqs. 19 and relations for $\hat{\mathcal{E}}_{\nu}^{(i)}$ similar to those of Eqs. (11), that the magnetic component of the incident beam then takes the form

$$
\begin{gathered}
\mathbf{H}_{p}(\mathbf{x} \mid \omega)_{i n c}=\int_{q_{\|}<\frac{\omega}{c}} d^{2} q_{\|} \hat{\mathcal{E}}_{s}^{(i)}\left(\mathbf{q}_{-}, \omega\right) \exp \left[i \mathbf{q}_{-} \cdot \mathbf{x}\right] \\
\times W\left(\mathbf{q}_{\|}, \mathbf{k}_{\|}\right),
\end{gathered}
$$

for a $p$-polarized beam, and

$$
\begin{gathered}
\mathbf{H}_{s}(\mathbf{x} \mid \omega)_{i n c}=-\int_{q_{\|}<\frac{w}{c}} d^{2} q_{\|} \\
\hat{\mathcal{E}}_{p}^{(i)}\left(\mathbf{q}_{-}, \omega\right) \exp \left[i \mathbf{q}_{-} \cdot \mathbf{x}\right] \\
\times W\left(\mathbf{q}_{\|}, \mathbf{k}_{\|}\right),
\end{gathered}
$$

for an $s$-polarized beam.

With the incident field in the form of Eqs. (21) and (22), the magnitude of the total time-averaged incident flux is the same for light of both polarizations, and is given by

$$
P_{i n c}^{(p, s)}=\frac{c^{2}}{8 \pi \omega} p_{i n c}
$$

where

$$
\begin{aligned}
p_{\text {inc }} & =w^{4} \int_{q_{\|}<\frac{\omega}{c}} d^{2} q_{\|} \alpha_{0}\left(q_{\|}\right) \exp \left[-w^{2}\left(\mathbf{q}_{\|}-\mathbf{k}_{\|}\right)^{2}\right] \\
& =2 \pi w^{4}\left(\frac{\omega}{c}\right)^{3} \exp \left(-w^{2} k_{\|}^{2}\right) \int_{0}^{\frac{\pi}{2}} d \theta \sin \theta \cos ^{2} \theta I_{0}\left(2 w^{2} \frac{\omega}{c} k_{\|} \sin \theta\right) \exp \left[-w^{2} \frac{\omega^{2}}{c^{2}} \sin ^{2} \theta\right],
\end{aligned}
$$


and $I_{0}(z)$ is the modified Bessel function of the first kind and zero order. In passing, it should be noted that in the large beam width limit, for which the beam approaches a plane wave, it follows from Eq. (24a) that $p_{\text {inc }}=S \alpha_{0}\left(k_{\|}\right)$ where $S$ is the area of the plane $x_{3}=0$ covered by the rough surface.

\section{Mean Differential Reflection Coefficient}

The differential reflection coefficient is defined as the fraction of the total time-averaged flux incident on the surface that is scattered into the element of solid angle $d \Omega_{s}$ about the scattering direction $\left(\theta_{s}, \phi_{s}\right)$. Since we are concerned with scattering from a randomly rough sur- face, it is the averaged (or mean) of this quantity over an ensemble of realizations of the surface that we need to calculate. From its definition, we find from Eqs. (14) and (23) that the mean differential reflection coefficient for the scattering of incident light of $\alpha$ polarization into light of $\beta$ polarization is given by

$$
\left\langle\frac{\partial R_{\beta \alpha}}{\partial \Omega_{s}}\right\rangle=\frac{1}{4 \pi^{2}}\left(\frac{\omega}{c}\right)^{3} \cos ^{2} \theta_{s} \frac{\left\langle\left|\mathcal{E}_{\beta}\left(\mathbf{q}_{+}, \omega\right)\right|^{2}\right\rangle}{p_{\text {inc }}} .
$$

If we write the scattering amplitude $\mathcal{E}_{\beta}\left(\mathbf{q}_{+}, \omega\right)$ as the sum of its mean value and the fluctuation about the mean,

$$
\mathcal{E}_{\beta}\left(\mathbf{q}_{+}, \omega\right)=\left\langle\mathcal{E}_{\beta}\left(\mathbf{q}_{+}, \omega\right)\right\rangle+\left[\mathcal{E}_{\beta}\left(\mathbf{q}_{+}, \omega\right)-\left\langle\mathcal{E}_{\beta}\left(\mathbf{q}_{+}, \omega\right)\right\rangle\right]
$$

each term contributes separately to the mean differential reflection coefficient

$$
\begin{aligned}
\left\langle\frac{\partial R_{\beta \alpha}}{\partial \Omega_{s}}\right\rangle & =\frac{1}{4 \pi^{2}}\left(\frac{\omega}{c}\right)^{3} \cos ^{2} \theta_{s} \frac{\left\langle\left|\mathcal{E}_{\beta}\left(\mathbf{q}_{+}, \omega\right)\right|^{2}\right\rangle}{p_{\text {inc }}} \\
& =\frac{1}{4 \pi^{2}}\left(\frac{\omega}{c}\right)^{3} \cos ^{2} \theta_{s} \frac{\left|\left\langle\mathcal{E}_{\beta}\left(\mathbf{q}_{+}, \omega\right)\right\rangle\right|^{2}}{p_{\text {inc }}}+\frac{1}{4 \pi^{2}}\left(\frac{\omega}{c}\right)^{3} \cos ^{2} \theta_{s} \frac{\left\langle\left|\mathcal{E}_{\beta}\left(\mathbf{q}_{+}, \omega\right)\right|^{2}\right\rangle-\left|\left\langle\mathcal{E}_{\beta}\left(\mathbf{q}_{+}, \omega\right)\right\rangle\right|^{2}}{p_{\text {inc }}}
\end{aligned}
$$

The first term in Eq. (27b) gives the contribution to the mean differential reflection coefficient from the light that has been scattered coherently,

$$
\left\langle\frac{\partial R_{\beta \alpha}}{\partial \Omega_{s}}\right\rangle_{c o h}=\frac{1}{4 \pi^{2}}\left(\frac{\omega}{c}\right)^{3} \cos ^{2} \theta_{s} \frac{\left|\left\langle\mathcal{E}_{\beta}\left(\mathbf{q}_{+}, \omega\right)\right\rangle\right|^{2}}{p_{i n c}} .
$$

The second term gives the contribution to the mean differential reflection coefficient from the light that has been scattered incoherently,

$$
\begin{aligned}
\left\langle\frac{\partial R_{\beta \alpha}}{\partial \Omega_{s}}\right\rangle_{\text {incoh }} & =\frac{1}{4 \pi^{2}}\left(\frac{\omega}{c}\right)^{3} \cos ^{2} \theta_{s} \\
\times & \frac{\left\langle\left|\mathcal{E}_{\beta}\left(\mathbf{q}_{+}, \omega\right)\right|^{2}\right\rangle-\left|\left\langle\mathcal{E}_{\beta}\left(\mathbf{q}_{+}, \omega\right)\right\rangle\right|^{2}}{p_{\text {inc }}} .
\end{aligned}
$$

The dependencies of the right-hand sides of these expressions on the polarization index $\alpha$ is through the dependence of the amplitudes $\mathcal{E}_{\beta}\left(\mathbf{q}_{+}, \omega\right)$ on the surface current $\mathbf{J}_{H}\left(\mathbf{x}_{\|} \mid \omega\right)$ in Eqs. 13). This surface current satisfies the inhomogeneous integral equations, Eqs. (7), in which the inhomogeneous terms depend on the incident field, and hence on its polarization $\alpha=p, s$. Thus $\mathcal{E}_{\beta}\left(\mathbf{q}_{+}, \omega\right)$ depends implicitly on the polarization $\alpha$ of the incident field, and so therefore does the differential reflection coefficient.

The procedure now is to generate a large number $N_{p}$ of realizations of the surface profile function $\zeta\left(\mathbf{x}_{\|}\right)$, and for each realization to solve the scattering problem for an incident field of $p$ or $s$ polarization. The solution is then used to calculate the scattering amplitude $\mathcal{E}_{\beta}\left(\mathbf{q}_{+}, \omega\right)$ and $\left|\mathcal{E}_{\beta}\left(\mathbf{q}_{+}, \omega\right)\right|^{2}$. An arithmetic average of the $N_{p}$ results for these quantities yields the quantities $\left|\left\langle\mathcal{E}_{p}\left(\mathbf{q}_{+}, \omega\right)\right\rangle\right|^{2}$ and $\left\langle\left|\mathcal{E}_{s}\left(\mathbf{q}_{+}, \omega\right)\right|^{2}\right\rangle$ entering Eqs. 28$)-(29)$ for the mean differential reflection coefficient.

\section{E. Energy conservation}

To facilitate the discussion of the conservation of energy, let us define the following quantity

$$
\mathcal{U}_{\alpha}^{\beta}\left(\theta_{0}, \phi_{0}\right)=\int d \Omega_{s}\left\langle\frac{\partial R_{\beta \alpha}}{\partial \Omega_{s}}\right\rangle .
$$

Recalling the definition of the mean differential reflection coefficient, it follows that the physical significance of 
$\mathcal{U}_{\alpha}^{\beta}\left(\theta_{0}, \phi_{0}\right)$ is that it is the fraction of the flux of the incident $\alpha$-polarized light that is scattered into $\beta$-polarized light by the rough surface irrespective of scattering direction.

For a perfectly conducting surface, all power flux incident onto the rough surface has to be converted into scattered power flux leaving the surface, since there is no absorption in the system. Hence, this is nothing but energy conservation, and it can be expressed in terms of $\mathcal{U}_{\alpha}^{\beta}\left(\theta_{0}, \phi_{0}\right)$ as

$$
\begin{aligned}
\mathcal{U}\left(\theta_{0}, \phi_{0}\right) & =\sum_{\alpha=p, s} \mathcal{U}_{\alpha}\left(\theta_{0}, \phi_{0}\right) \\
& =\sum_{\beta=p, s} \sum_{\alpha=p, s} \mathcal{U}_{\alpha}^{\beta}\left(\theta_{0}, \phi_{0}\right) \\
& =1,
\end{aligned}
$$

where the $\alpha$-summation over the polarization of the incident light is only non-trivial in cases where the incident beam does not have a well-defined $p$ - or $s$-polarization. It was pointed out in the previous subsection, that the mean differential reflection coefficient can be separated into a coherent and an incoherent component. The same applies therefore to $\mathcal{U}\left(\left(\theta_{0}, \phi_{0}\right)\right.$ and related quantities.

We note that Eq. (31) is rather useful for estimating the quality of the simulations, including making sure that the discretization interval is fine enough. However, it should be stressed that relation (31) is only a necessary condition, and its satisfaction does not guarantee that the simulations are correct.

\section{RESULTS AND DISCUSSIONS}

We have carried out calculations of the scattering of $p$ - and $s$-polarized light from a randomly rough perfectly conducting surface with an rms height $\delta=\lambda$ and a transverse correlation length $a=2 \lambda$, where $\lambda$ is the wavelength of the incident field. The polar angles of incidence are $\theta_{0}=0^{\circ}, 20^{\circ}$ and $40^{\circ}$, while the azimuthal angle of incidence in all cases is $\phi_{0}=0^{\circ}$. The surface is generated at a $112 \times 112$ grid of points covering an area $L^{2}=16 \lambda \times 16 \lambda$. The integration mesh size is therefore $\Delta x=\lambda / 7$. The calculations were carried out for an incident field in the form of a Gaussian beam [Eqs. [20)] of width $w=4 \lambda$.

In Fig. 2 we plot the mean differential reflection coefficients as functions of the polar scattering angle $\theta_{s}$ for the in-plane $\left(\phi_{s}=0^{\circ}\right)$ and out-of-plane $\left(\phi_{s}= \pm 90^{\circ}\right)$, co$(p \rightarrow p)$ and cross- $(p \rightarrow s)$ polarized scattered light due to a $p$-polarized Gaussian beam incident on the surface. The results depicted in Figs. 2 were obtained as averages over 12,000 realizations of the surface profile function. In obtaining these results we have noted that at least for the roughness parameters we have assumed, the contribution to the mean differential reflection coefficient from the light scattered coherently is smaller than the contri-

\begin{tabular}{ccccccc}
\hline \hline$\theta_{0}[\mathrm{deg}]$ & $\alpha$ & $\mathcal{U}$ & $\mathcal{U}_{\text {incoh }}$ & $\mathcal{U}_{\text {coh }}\left[10^{-4}\right]$ & $\mathcal{U}_{\alpha}^{p} / \mathcal{U}$ & $\mathcal{U}_{\alpha}^{s} / \mathcal{U}$ \\
\hline 0 & $p$ & 0.9976 & 0.9975 & 0.9 & 0.5054 & 0.4946 \\
20 & $p$ & 0.9962 & 0.9961 & 0.9 & 0.5315 & 0.4686 \\
40 & $p$ & 0.9951 & 0.9947 & 3.8 & 0.5407 & 0.4592 \\
0 & $s$ & 0.9970 & 0.9967 & 3.1 & 0.5021 & 0.4979 \\
20 & $s$ & 0.9966 & 0.9963 & 2.8 & 0.4939 & 0.5061 \\
40 & $s$ & 0.9953 & 0.9948 & 4.9 & 0.4834 & 0.5166 \\
\hline \hline
\end{tabular}

TABLE I: The energy conservation for various polar angles of incidence $\left(\theta_{0}\right)$ and incidence polarizations $(\alpha)$ for the surface parameters given in the text. The surface and scattering amplitude were discretized on $112 \times 112$ and $101 \times 101$ grids, respectively. These results were obtained on the basis of Eqs. 30 and (31).

bution from the light scattered incoherently by a factor of approximately $10^{-4}$ (see Table I for details).

There is no single scattering contribution in the cases of in-plane cross-polarized [Fig. 2(b)] and out-of-plane co-polarized [Fig. 2(c)] scattering. This we believe is the main reason for the reduced amplitude of the mean differential reflection coefficients in these cases relative to those of Fig. 2(a) and (d) where single scattering is allowed. The peaks at $\theta_{s}=0^{\circ}$ and $-20^{\circ}$ [20] for inplane co-polarized scattering [Figs. 2(a)] are enhanced backscattering peaks [16, 17, 18, 19]. However, the structures seen as peaks in the backscattering directions of the cross-polarized scattering, Fig. 2(b), are not real peaks, as will be seen below from the full angular intensity distributions. The results that the mean differential reflection coefficients for out-of-plane co- and cross-polarized scattering [Figs. 2(c) and (d)] are even functions of $\theta_{s}$ are consequences of the scattering geometry, namely that $\phi_{0}=0^{\circ}, \phi_{s}= \pm 90^{\circ}$, and the isotropy of the power spectrum of the surface roughness.

In Fig. 3 we present corresponding results to those of Fig. 3, but now for an $s$-polarized incident Gaussian beam. There is no single scattering contribution to the in-plane cross-polarized and out-of-plane co-polarized scattering, as in the case of $p$ polarization. Also in this case the peaks seen in the in-plane co-polarized scattering [Fig. 3(a)] are enhanced backscattering peaks, while the structures seen in the in-plane cross-polarized scattering [Fig. 3(b)] in the backscattering direction are not real peaks.

The full angular distribution of the intensity of the scattered light is presented as color contour plots in Figs. 4 6, which correspond to the polar angles of incidence $\theta_{0}=0^{\circ}, 20^{\circ}$, and $40^{\circ}$, respectively, and for several combinations of the polarizations of the incident and scattered light 21]. To the best of our knowledge, this is the first time that the full angular distributions of the light scattered from a strongly rough surface have been obtained by a rigorous computer simulation approach. It is observed from Figs. 46 that the angular distributions, for given polarizations of the incident and scattered light, are far from trivial, and show strong and complex 

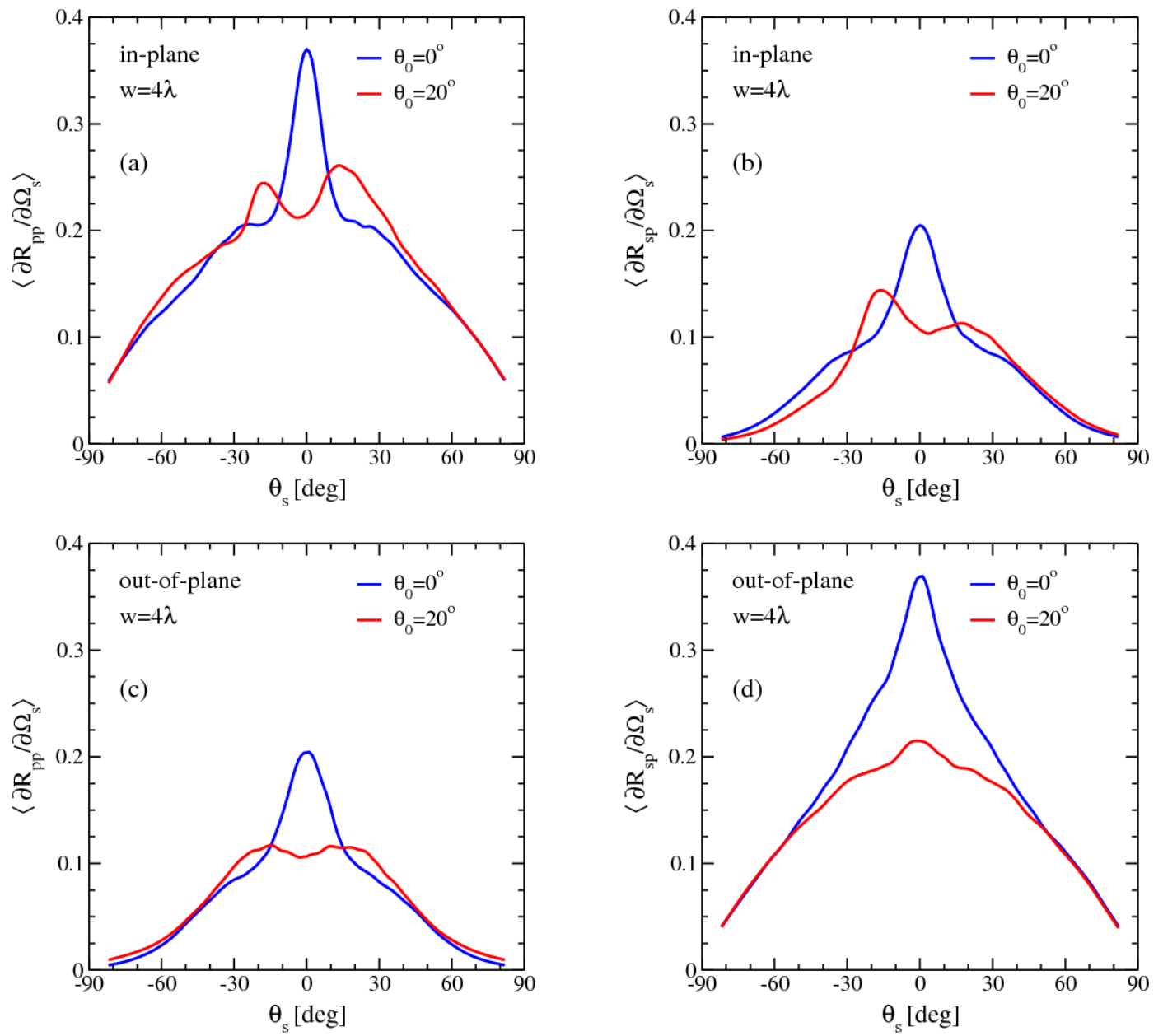

FIG. 2: (Color online) The mean differential reflection coefficients, $\left\langle\partial R_{\beta \alpha} / \partial \Omega_{s}\right\rangle(\alpha \rightarrow \beta)$, as functions of the polar scattering angle $\theta_{s}$ for the in-plane $\left(\phi_{s}=\phi_{0}\right.$ or $\left.\phi_{s}=\phi_{0}+180^{\circ}\right)$ and out-of-plane $\left(\phi_{s}=\phi_{0} \pm 90^{\circ}\right)$, co- $(p \rightarrow p)$ and cross- $(p \rightarrow s)$ polarized scattering of a $p$-polarized incident beam $(\alpha=p)$ of width $w=4 \lambda\left(\theta_{0}=0^{\circ}\right.$ and $\left.\theta_{0}=20^{\circ} ; \phi_{0}=0^{\circ}\right)$ scattered from a Gaussian randomly rough perfectly conducting surface. The Gaussian correlated surface had a correlation length $a=2 \lambda$ and an rms height $\delta=\lambda$. To facilitate comparison between the various configurations presented in this figure, notice that we have used similar scales for all ordinate axes. Moreover, to simplify the presentation of the figures, a convention was adopted where negative (positive) values of $\theta_{s}$ correspond to $\phi_{s}=\phi_{0}+180^{\circ}\left(\phi_{s}=\phi_{0}\right)$.

angular dependencies. With the full angular dependence of the scattered light available, the energy conservation of the simulations performed can be obtained by comparing the power incident on the surface to that being scattered from it [see Eq. [31)]. For normal incidence, we obtained $\mathcal{U}_{p}=0.9976$ and $\mathcal{U}_{s}=0.9970$ for $p$ - and $s$-polarized incident light, respectively. For the other angles of incidence considered, $\theta_{0}=20^{\circ}$ and $40^{\circ}$, energy conservation was satisfied within $0.5 \%$ or better (see Table I for details). Even if energy conservation is only a necessary requirement, such results, however, still testify to the accuracy of the simulations and the approaches used to obtain them.

It is interesting to note that for the roughness parameters considered, the power in a normally incident beam is divided essentially equally between $p$ and $s$ polarized scattered light (independent of the polarization of the in- cident light). This effect we attribute to multiple scattering. For the other angles of incidence, it is observed from Table I that the fraction of incident power being scattered into the same polarization as that of the incident beam (co-polarized scattering), but still independent of scattering direction, increases with the polar angle of incidence.

We will now discuss Figs. 46 in more detail: We start by considering the case of normal incidence; $\theta_{0}=0^{\circ}$ and $\phi_{0}=0^{\circ}$ [Figs. 4]. Recall that with the assumptions and conventions used in this work, the electric field of an incident $p$-polarized Gaussian beam is in the plane of incidence. In Fig. 4(a) we present a contour plot of the mean differential reflection coefficient for the scattering of $p$-polarized light into either $p$ - or $s$-polarized scattered light, (i.e. the polarization state of the scatted light is not being recorded). The angle-dependent scattering, in 

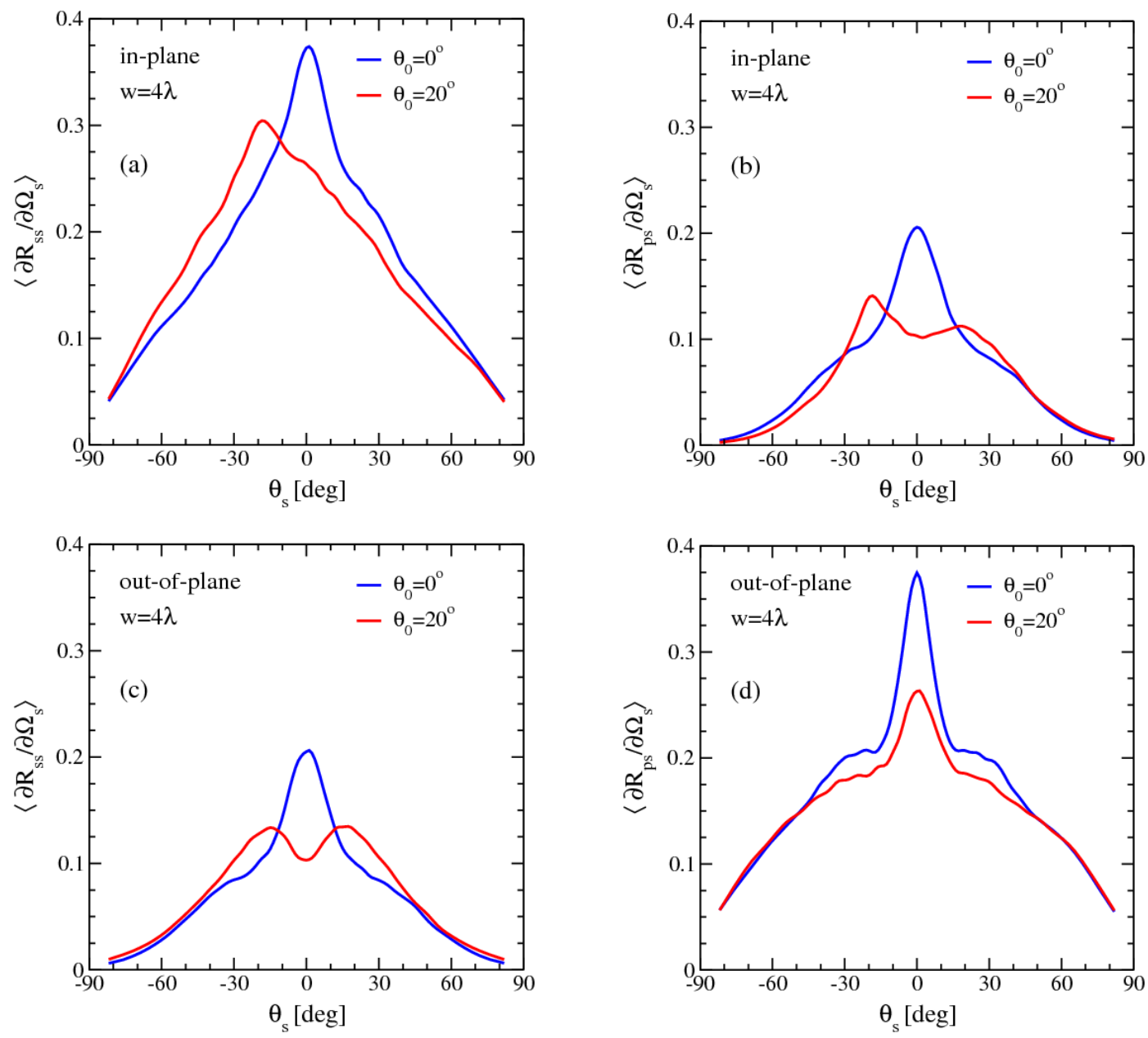

FIG. 3: Same as Fig. 2, but for an s-polarized incident beam.

this case, is for the most part rather isotropic, except for a slight anisotropy seen as an elongated (along the $q_{2}$-direction) structure around the normal scattering direction. This structure is caused by the wider intensity distribution in the direction perpendicular to the incident electric field as compared to the intensity distribution along it. The central peak present in Fig. 4(a) is the enhanced backscattering peak, and is not related to specular scattering which for these roughness parameters can be neglected (see Table $\mathrm{I}$ for details). A similar behavior is seen for the scattering of (normally) incident $s$-polarized light into either $p$ - or $s$-polarized light [Fig. 4(b)]. Here an apparent enhanced backscattering peak is also observed. In the case of $s$-polarization, one sees though that the central anisotropic portion of the scattering has a different orientation compared to that in the case of $p$-polarization. It remains true, however, that there is a stronger scattering perpendicular to the (average) direction of the incident electric field independent of the polarization of the incident light.

Based on these findings, one may be misled into believing that the scattering for normal incidence into the two possible (linear) polarizations, $p$ or $s$, is also more-orless isotropic, except maybe for some minor polarization dependence for the smaller scattering angles $\theta_{s}$. However, this is rather far from being true. In Figs. 4(c) and (d) we present the scattering into $p$-polarized scattered light from, respectively, a (normally) incident $p$ and $s$ polarized Gaussian beam. Similarly, depicted in Figs. 4(e) and (f) are the scattering into $s$-polarized scattered waves for an incident $p$ - or $s$-polarized Gaussian beam. We note that taking the sum of the distributions shown in e.g. Figs. 4(c) and (e) produces the angular distribution shown in Fig. 4(a). From Figs. 4(c)-(f) it follows that the intensity distributions for scattering from one polarization into another, or into the same one, show a dipole-like angular dependence.

For co-polarized scattering, i.e. the polarization of the incident light and the (recorded) polarization of the scattered light are the same, the "forward direction" of the dipole-like pattern is oriented along $q_{1}$ [Figs. 4(c) and (f)], while for cross-polarization, it is oriented along the $q_{2}$ direction [22]. For normal incidence, the $\mathbf{k}$-vector used to define the incident Gaussian beam, does not 

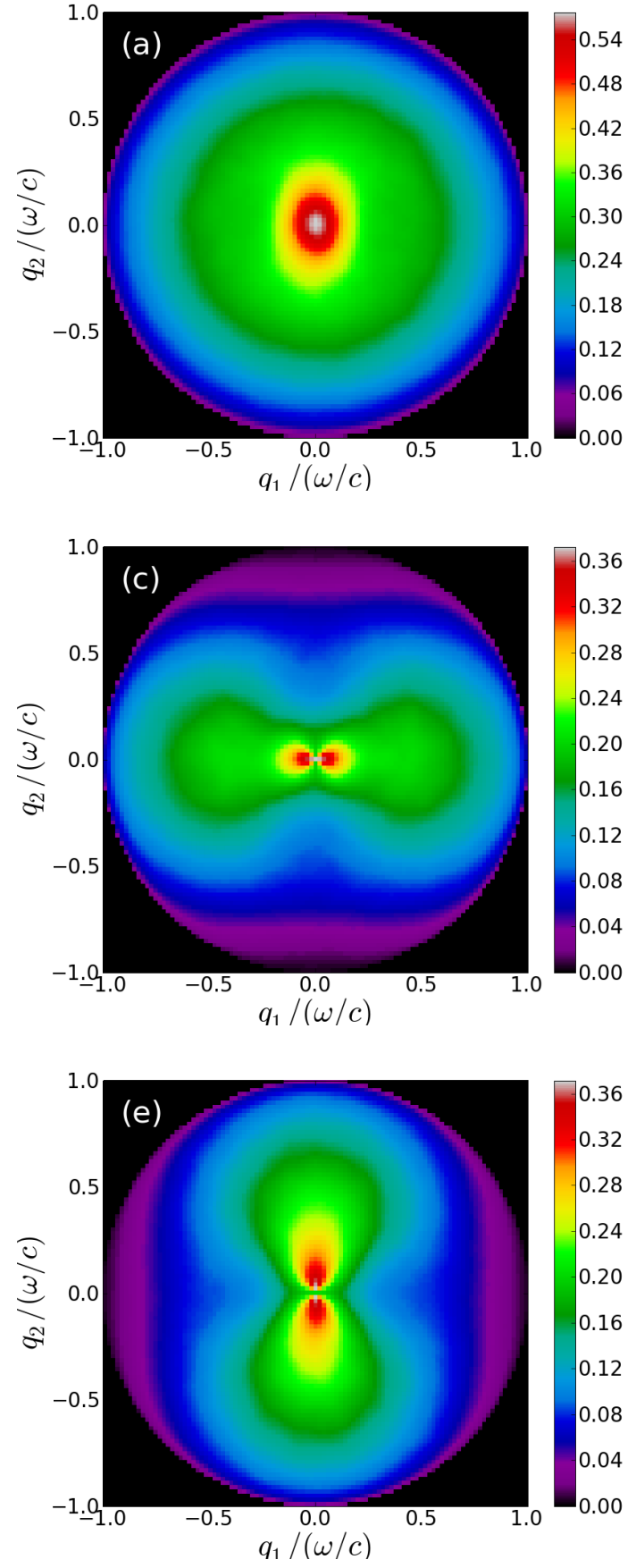
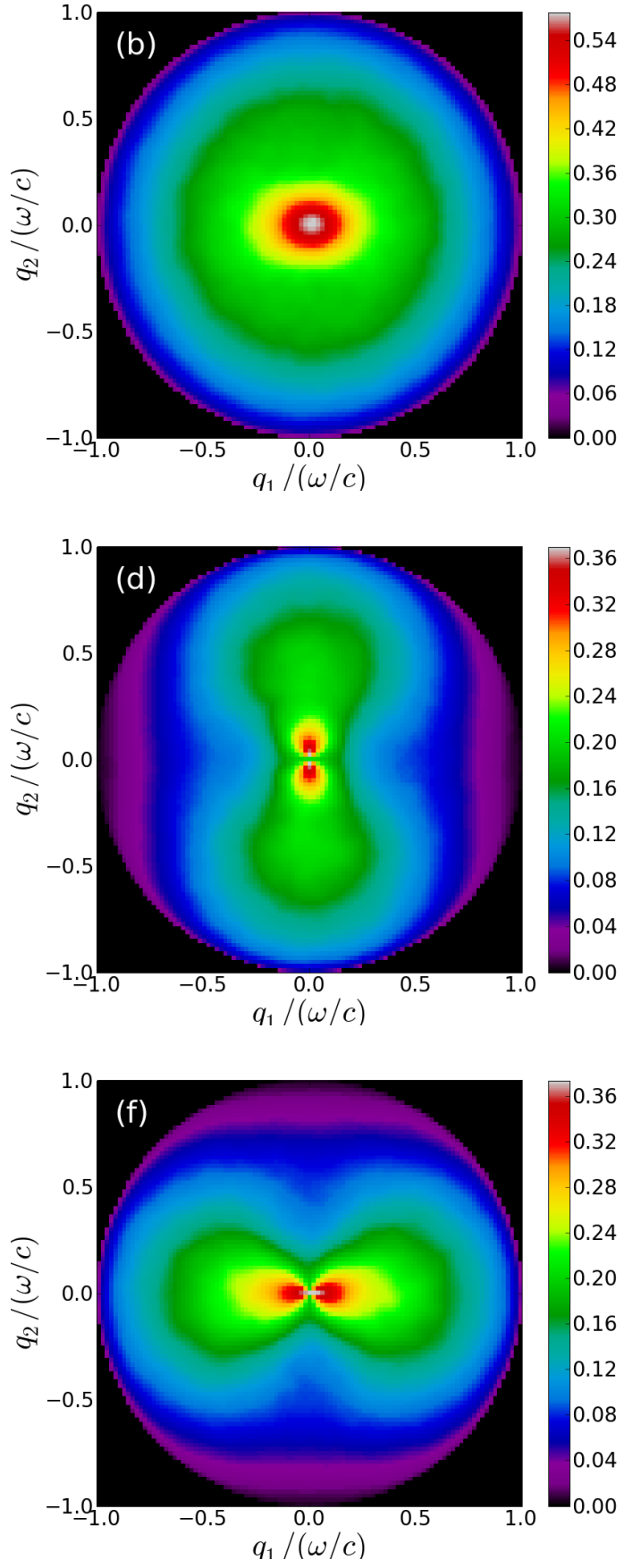

FIG. 4: (Color online) The complete angular distributions of the mean differential reflection coefficient, $\left\langle\partial R_{\beta \alpha} / \partial \Omega_{s}\right\rangle$, for the scattering of an $\alpha$-polarized Gaussian beam incident on the surface at polar angle $\theta_{0}=0^{\circ}$ and azimuthal angle $\phi_{0}=0^{\circ}$. The perfectly conducing rough surface was characterized by a Gaussian height distribution of rms-value $\delta=\lambda$ and a Gaussian correlation function of transverse correlation length $a=2 \lambda$. The incident beam was $p$-polarized in Figs. 4(a), (c) and (e) [left column]; and $s$-polarized in Figs. 4(b), (d) and (f) [right column]. Moreover, in the top two figures [Figs. 4(a) and (b)] the polarization of the scattered light was not recorded; in Figs. 4(c) and (d) [central row] only p-polarized scattered light was recorded; while the bottom two figures correspond to recording only s-polarized scattered light [Figs. 4(e) and (f)]. The rough surface, covering an area $16 \lambda \times 16 \lambda$, was discretized at a grid of $112 \times 112$ points corresponding to a distcretization interval $\lambda / 7$ for both directions. The presented figures were obtained by averaging the mean differential reflection coefficient over 12,000 surface realizations. 

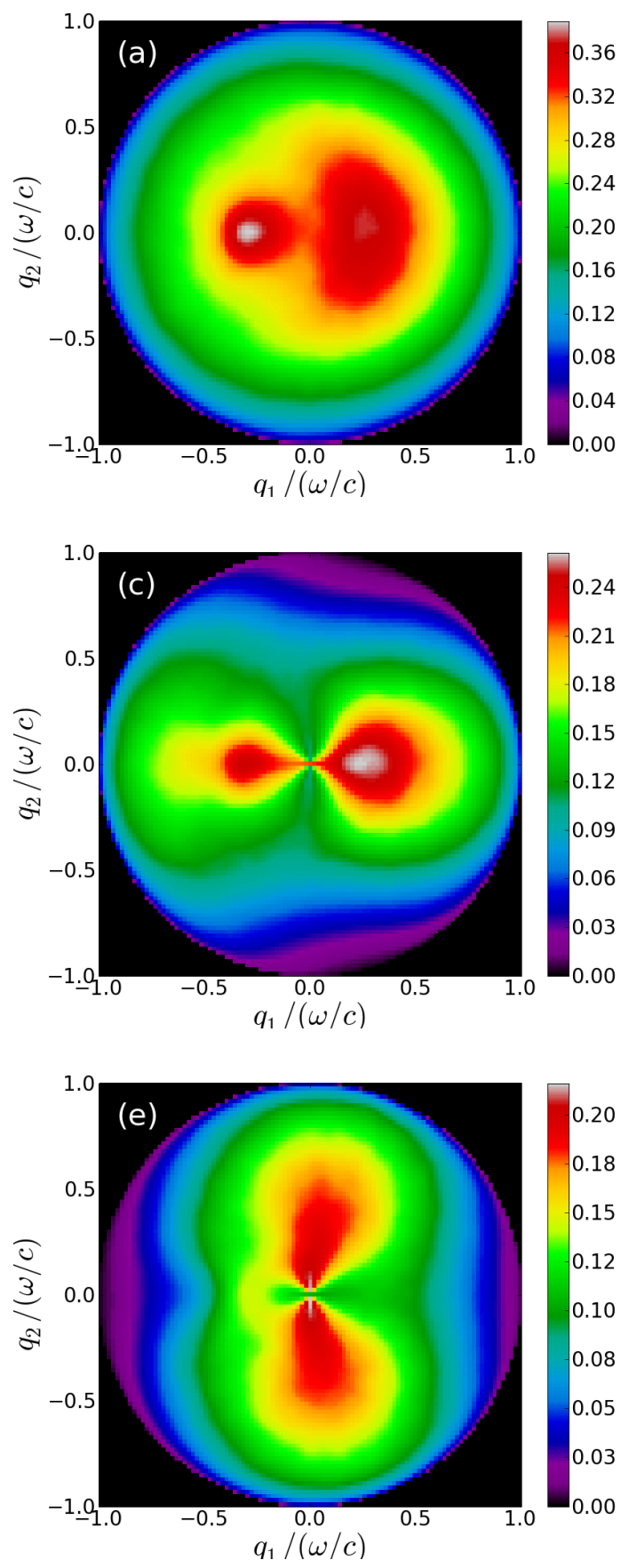
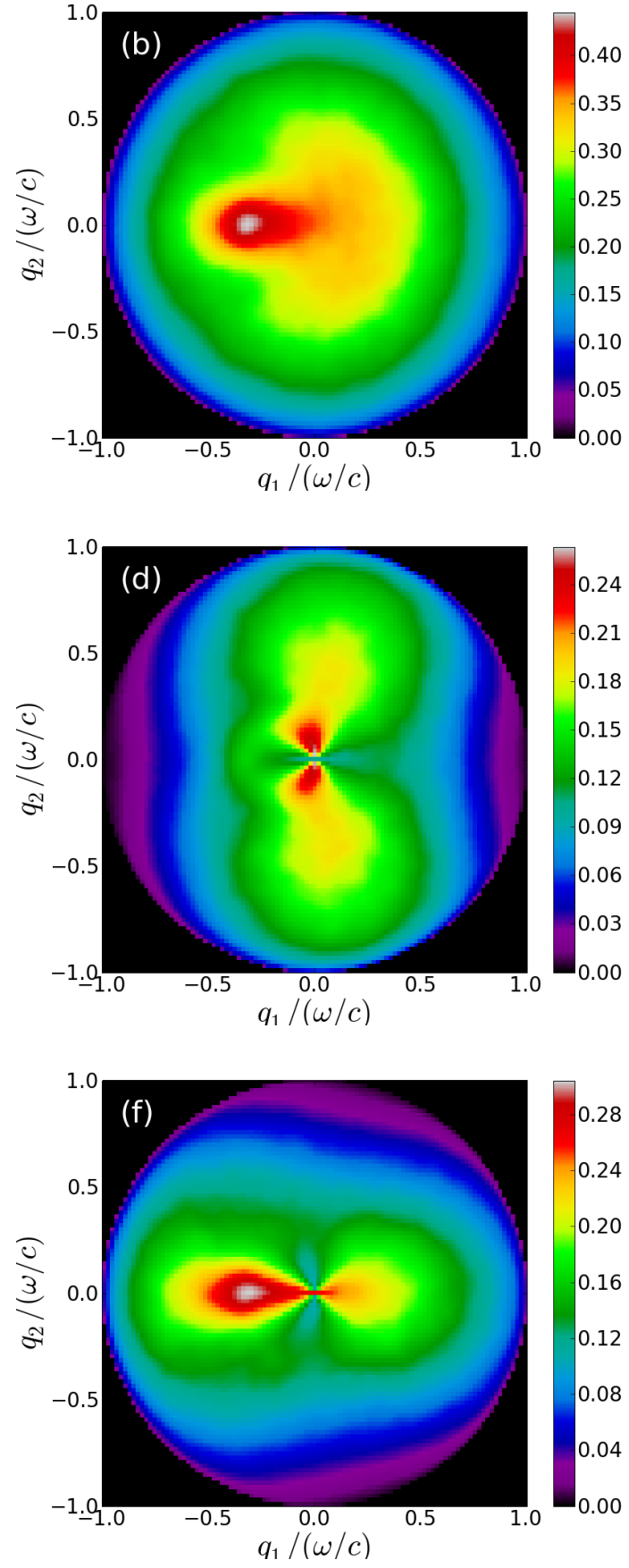

FIG. 5: (Color online) Same as Figs. 4 but for a polar angle of incidence $\theta_{0}=20^{\circ}$.

(together with $\hat{\mathbf{x}}_{3}$ ) define a plane of incidence. However, we have used the convention in the simulations, that the plane of incidence is defined as the plane having $\hat{\boldsymbol{\phi}}_{0}=-\sin \phi_{0} \hat{\mathbf{q}}_{1}+\cos \phi_{0} \hat{\mathbf{q}}_{2}$ as its normal vector which is well-defined for all polar angles of incidence (also $\theta_{0}=0^{\circ}$ ) and coincides with the usual definition when $\theta_{0} \neq 0$. Since $\phi_{0}=0^{\circ}$ was assumed for all the simulation results presented, it follows (with this convention) that the plane of incidence is the $q_{1} q_{3}$-plane. With this definition for the plane of incidence, we may rephrase the above observation: For co- and cross-polarized scattering the dipole-like pattern is oriented along and perpendicular to the plane of incidence, respectively. Later we will see that this statement also holds true for non-normal incidence.

It is noted that we have checked and found that the scattering of a normally incident unpolarized beam by 

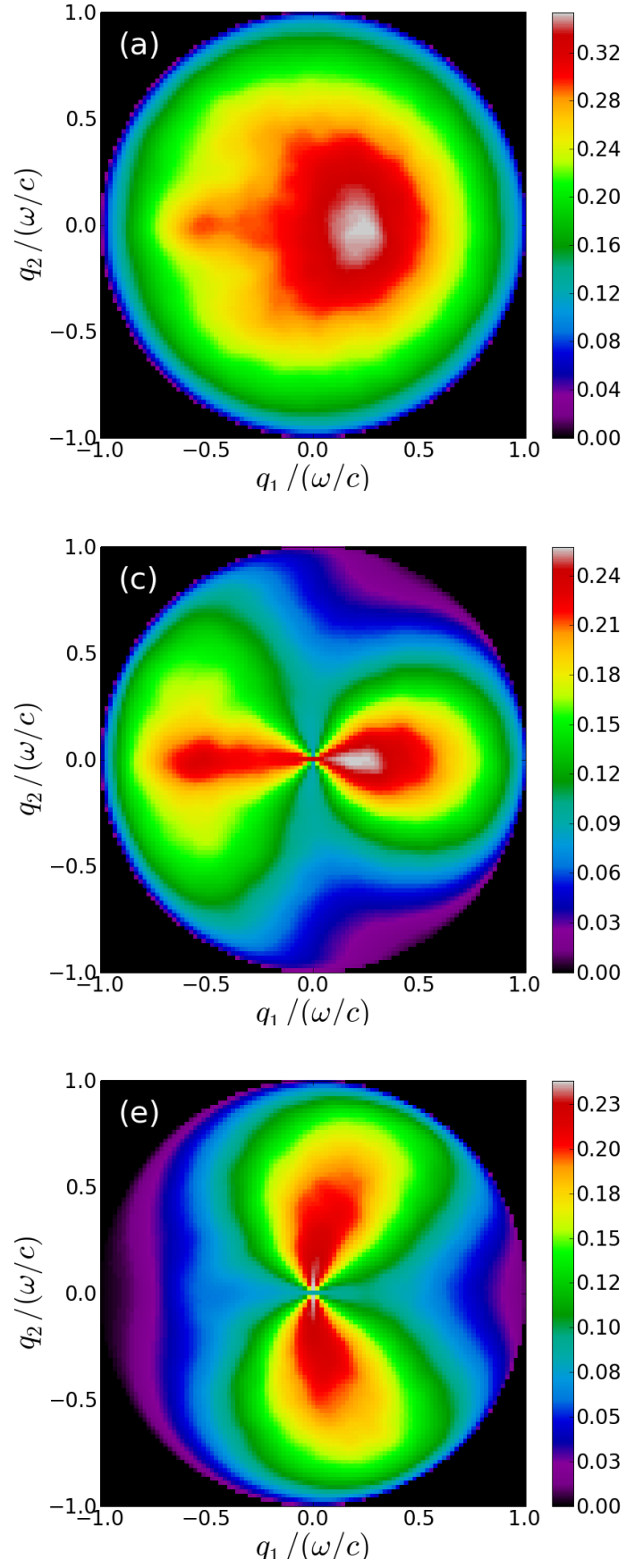
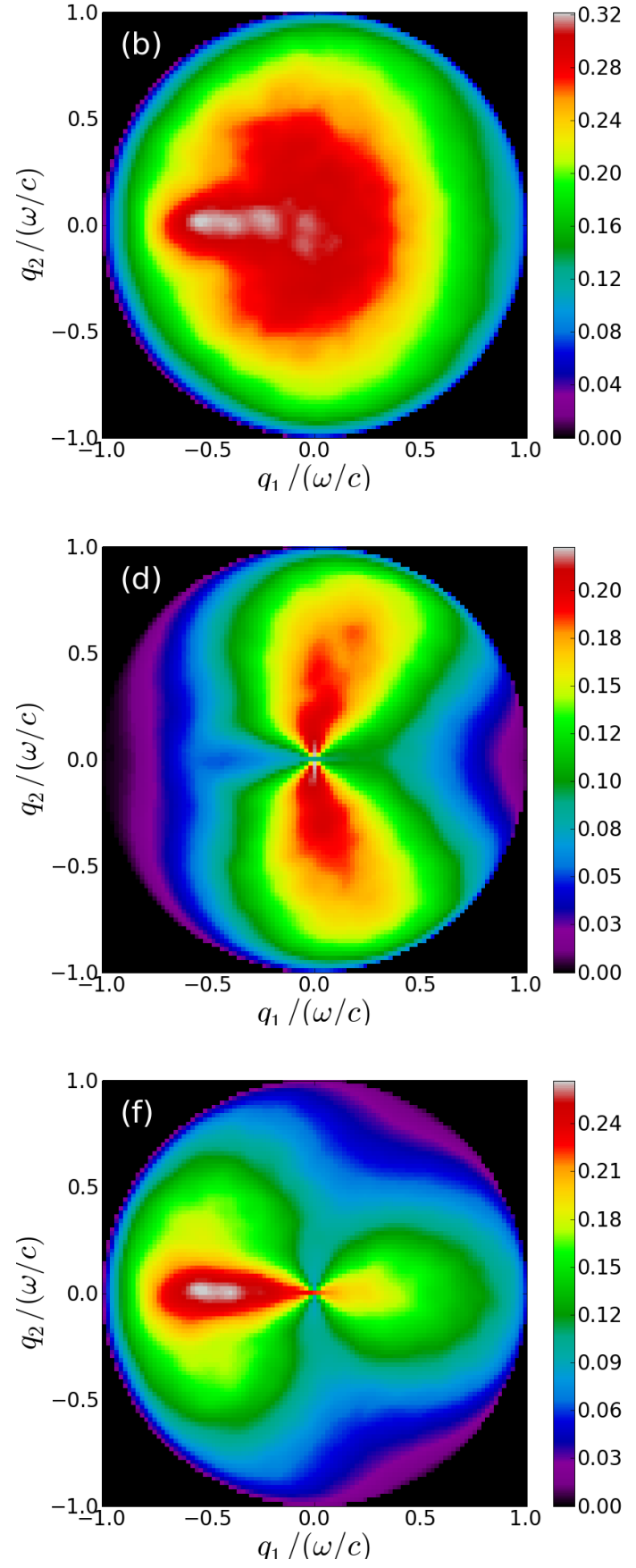

FIG. 6: (Color online) Same as Figs. 4 but for a polar angle of incidence $\theta_{0}=40^{\circ}$.

the rough surface, produces, when both its $p$ - and $s$ polarized components are recorded, a fully rotationally symmetric intensity distribution (equal to the sum of the distributions in Figs. 4(a) and (b)). If only $p$ - or $s$-polarized scattered light is recorded, one will still, with the same type of unpolarized illumination, obtain rotationally symmetric intensity distributions (equal to the sum of the distributions from Figs. 4(c) and (d), in the case of $p$-polarization, and the sum of Figs. $4(\mathrm{e})$ and (f) for $s$-polarization).

We now turn our attention to the scattering for nonnormal incidence. In Figs. 5 we present the results for the angular distribution of the mean differential reflection coefficient for either a $p$ - or $s$-polarized Gaussian beam incident onto the surface at a polar angle $\theta_{0}=20^{\circ}$ and scattered into various polarization states. 
From Figs. 5(a) and (b), for which the polarization of the scattered light is not recorded, one observes that there are pronounced enhanced backscattering peaks located around the backscattering direction (at $\theta_{s}=20^{\circ}$ and $\left.\phi_{s}=180^{\circ}\right)$. It is also observed that the $p$-polarized incident beam tends to scatter more light into the forward plane $\left(q_{1}>0\right)$ than does an $s$-polarized incident beam.

The first thing to notice from Figs. 5(c)-(f), where the polarization of the scattered light is recorded, is that the co-polarized scattering shows up as an elongated structure with the long axis of the pattern directed along the plane of incidence, while the cross-polarized scattering has the long axis of the scattering pattern perpendicular to this plane. This observation is in agreement with what was already observed above for normal incidence. However, for non-normal incidence, the patterns do show less symmetry, as expected, and an even richer and more complicated angular structure. In principle, the enhanced backscattering peak phenomenon should exist in both coand cross-polarized scattering [17, 18, 19. However, for the roughness parameters assumed in this work, one observes instead of a well-pronounced peak in the backscattering direction, a ridge of constant enhanced intensity in parts of the backscattering plane $\left(q_{1}<0\right)$ forming (what seems to be) a half circle of constant polar scattering angle $\theta_{s} \approx \theta_{0}=20^{\circ}$ with $\phi_{s} \in\left[90^{\circ}, 270^{\circ}\right.$ ] [Figs. 5(d) and (e)]. In exactly the backscattering direction, $\theta_{s}=\theta_{0}$ and $\phi_{s}=180^{\circ}$, there seems to be little, if any, "extra" enhancement in the cross-polarized scattering as compared to the intensities at other values of $\phi_{s}$ in the interval $\left[90^{\circ}, 270^{\circ}\right]$. The enhancement ridge seen is Figs. 5(d) and (e) we speculate is caused by a constructive interference effect similar in nature to the underlying enhanced backscattering.

In passing, we note that having available only the inplane and out-of-plane results for the same angle of incidence, the local enhancements observed in e.g. Figs. 2(b) and 3 (b) for $\theta_{0}=20^{\circ}$, could easily have been mistaken for well-localized features in the backscattering direction, similar to what one has for co-polarized scattering [Figs. 5(c) and (f)]. In this respect, the angular intensity distributions of the kind presented in Figs. 46 can provide important contributions to a better understanding of the multiple scattering phenomena.

Figures 6 present contour plots of the angular distributions of the mean differential reflection coefficient for a polar angle of incidence $\theta_{0}=40^{\circ}$. Since these results rather closely resembles those of Figs. 5, we will not discuss them further. However, we note that the structures due to coherent interference seen in the cross-polarized components for $\theta_{0}=20^{\circ}$, are much harder to identify in the results for $\theta_{0}=40^{\circ}$. This is believed to be caused by the relatively large angle of incidence, for which it is known that coherent effects become weaker 16 .

\section{NUMERICAL ASPECTS}

\begin{tabular}{|c|c|c|c|c|c|c|c|}
\hline \multirow[t]{3}{*}{$N$} & \multirow[t]{2}{*}{$\overline{t_{t o t}[\mathrm{~s}]}$} & \multirow[t]{2}{*}{$\overline{t_{A}[\mathrm{~s}]}$} & \multicolumn{2}{|c|}{$t_{A \mathbf{x}=\mathbf{b}}[\mathrm{s}]$} & \multirow[t]{2}{*}{$\overline{t_{\mathcal{E}}[\mathrm{s}]}$} & \multirow[t]{2}{*}{$\overline{\overline{\mathcal{N}}}$} & \multirow[t]{2}{*}{$\overline{\overline{\mathcal{M}_{A}}[\mathrm{~Gb}]}$} \\
\hline & & & $\overline{\text { BiCGStab }}$ & $\overline{\mathrm{LU}}$ & & & \\
\hline & 10.5 & 4.0 & 3.5 & 127 & 3.0 & 8192 & 0.50 \\
\hline 80 & 22.0 & 9.5 & 8.0 & 474 & 4.5 & 12800 & 1.22 \\
\hline 100 & 58.5 & 23.0 & 28.5 & 1780 & 7.0 & 20000 & 2.98 \\
\hline 112 & 76.0 & 36.0 & 31.0 & 3540 & 9.0 & 25088 & 4.69 \\
\hline
\end{tabular}

TABLE II: The CPU time spent on various stages of the calculations for one realization of the surface profile function and one angle of incidence. All CPU times are measured in seconds, and the numbers have been rounded to the closest half second, and they refer to a machine running an Intel Core2 CPU (Q9550) operating at $2.83 \mathrm{GHz}$ and running the Linux operating system. The surface was discretized on a $N \times N$ grid of points. The reported CPU times are: the total CPU time spent for simulating one surface realization for one angle of incidence including reading and writing of data $\left(t_{t o t}\right)$; the setup of the system matrix of the linear system $A \mathbf{x}=\mathbf{b}$ determining the surface currents $\left(t_{A}\right)$; the time to solve this system by a the iterative BiCGStab method or the direct LU decomposition method $\left(t_{A \mathbf{x}=\mathbf{b}}\right)$; and finally the time to calculate the reflection amplitudes, $\mathcal{E}\left(\mathbf{q}_{+}, \omega\right)$ for both scattered polarizations on a grid of $101 \times 101$ points $\left(t_{\mathcal{E}}\right)$. The number of unknowns to be solved for is $\mathcal{N}=2 N^{2}$, where the memory (in Gigabytes $(\mathrm{Gb})$ ) required to hold the complex system matrix $A$, using single precision, is denoted by $\mathcal{M}_{A} \propto \mathcal{N}^{2}=4 N^{4}$.

The rigorous computer simulation approach presented in this work is rather computationally demanding. Therefore, it is important to be able to perform such simulations in an efficient manner. One of the most challenging aspects of implementing a surface integral method for a two-dimensional rough surface, is the memory requirement. By discretizing the relevant integral equations, in this case Eq. (4), they are converted into a linear system $A \mathbf{x}=\mathbf{b}$, where $A$ denotes a dense complex system matrix; $\mathbf{b}$ is the right-hand-side given in terms of the incident field; and the unknown vector to be solved for, $\mathbf{x}$, consists (in our case) of the independent components of the surface current $\mathbf{J}_{H}\left(\mathbf{x}_{\|} \mid \omega\right)$. If the randomly rough surface $x_{3}=\zeta\left(\mathbf{x}_{\|}\right)$is discretized into $N \times N$ points, then the number of unknowns would be $\mathcal{N}=2 N^{2}$, since for a perfectly conducting rough surface we have two unknowns per surface point (the two independent components of $\left.\mathbf{J}_{H}\right)$. Hence, the amount of memory needed to hold the (full) system matrix of the scattering from a perfectly conducting surface is $\mathcal{M}_{A}=4 N^{4} m$, where $m$ is the size of a single scalar complex variable, which on most systems for single and double precision, respectively, is $m_{S}=8$ bytes and $m_{D}=16$ bytes.

For each surface realization, there are essentially three time-consuming steps in this kind of simulation. They are: $(i)$ to set up the system matrix; $(i i)$ to solve the linear system for the unknown surface currents; and (iii) to calculate the reflection amplitudes. Of the three, it is primarily the first two that are critical and, if not handled 
properly, particularly the second. For instance, the total CPU time taken to complete the calculation using single precision and an iterative solver for one angle of incidence and one surface realization with $N=112$, including reading input and writing output data, is $t_{t o t}=76.0 \mathrm{~s}$ on an Intel Core2 CPU (Q9550) operating at $2.83 \mathrm{GHz}$ and running the Linux operating system. On the other hand, for the same simulation the three steps mentioned above take $t_{A}=36.0 \mathrm{~s}$ to set up the system matrix, $t_{A x=b}=31.1 \mathrm{~s}$ to solve the linear system by the use of the iterative BiCGStab method, and $t_{\mathcal{E}}=8.9 \mathrm{~s}$ to calculate the reflection amplitudes on a $101 \times 101$ grid, in total $76.0 \mathrm{~s}$. Hence, the additional steps of the calculation, like generating the surface, reading and writing data to file etc., contribute only insignificantly to the overall CPU time $(t \sim 0.05 \mathrm{~s})$. The computation times for other surface discretizations are summarized in Table [II. The reason that it takes a relatively long time (compared to $t_{t o t}$ ) to set up the matrix elements is the cost of calculating the exponential function contained in the Green's function.

However, the most critical point to address when trying to reduce the overall CPU time, is the method used to solve the linear system. In this work, an iterative solver known as the stabilized bi-conjugated gradient method (BiCGStab) [10] has been used, and found to perform well and to produce reliable results for our application. The iteration process of the BiCGStab solver (using a Jacobi preconditioner) was terminated when the relative error was $10^{-5}$ (or less), which for normal incidence and with $N=112$ required typically a little more then 20 iterations when starting from an initial guess $\mathbf{x}_{\text {guess }}=0$ (of course, other surface parameters and initial guesses may require more or fewer iterations in order to reach the desired accuracy). Using a direct solver, like the LUdecomposition, would have taken significantly longer (see Table II). For instance, the time taken to solve the linear system for $N=112$ by a direct LU solver is 114 times longer than that taken by the BiCGStab solver (TableII). Moreover, this difference is expected to increase with increasing $N$ due to the different scaling with the number of unknowns (as also shown by the times presented in Table II). It should be noted that a direct solver, like the LU-decomposition, opens the possibility for carrying out calculations for several angles of incidence (the right-hand sides of the system) simultaneously with little addition to the overall computation time. This is not the case for the BiCGStab-method, where the solution time for several angles of incidence scales linearly with the number of angles of incidence. There are, however, other iterative methods that can solve a linear system with several right-hand-sides with only moderate increase in computational times. One such method is the (restarted) Generalized Minimal Residual Method (GMRES) method [14. Compared to the BiCGStab used here, the GMRES is typically more memory demanding and, therefore, this possibility has not been explored in this work.

For the sake of comparison, we have repeated the calculations reported by Tran and Maradudin in Ref. [1] using the same numerical parameters (the surface roughness parameters were already the same). For the calculations carried out in Ref. 1] solving the integral equations on a grid of $64 \times 64$ surface points, each iteration (of which there were six) required $365 \mathrm{CPU}$ seconds (on a Cray XMP/EA-116 machine), and to calculate the scattered fields, in-plane or out-of-plane, required $360 \mathrm{CPU}$ seconds for each realization of the surface profile function, for a total of $2550 \mathrm{CPU}$ seconds for each realization of the surface profile function. A similar calculation required only 7.6 CPU seconds per surface realization, a dramatic improvement in speed 23. This dramatic reduction occurred for two reasons: First, we have the overall improvement in general computer hardware. Second, we hold the whole system matrix in memory due to sufficient memory, while the approach used in Ref. 1 was to regenerate the matrix elements as they were needed. This time cost of the latter is not insignificant, as we can see from Table II. and both factors contribute to the overall speedup.

\section{CONCLUSIONS}

In conclusion, we have shown that the use of the method of moments and the biconjugate gradient stabilized method provides a formally exact solution to the problem of the scattering of an electromagnetic field from a two-dimensional, randomly rough, perfectly conducting surface, with a modest expenditure of computational time.

Moreover, the full angular distribution of the intensity of the scattered light, both co- and cross-polarized, was obtained by a formally rigorous approach for a strongly rough surface. Such distributions can display rather complex angular patterns that are rooted in the multiple scattering processes taking place when light interacts with a strongly rough surface.

Due to the full angular intensity distribution being accessible, the conservation of energy was checked explicitly for all the calculations reported and found to be satisfied with an error smaller than $0.5 \%$, or better, something that testifies to the accuracy of the approach and a satisfactory discretization.

\section{Acknowledgments}

This research was supported in part by AFRL contract FA9453-08-C-0230. The research of I.S. was in addition supported in part by the Research Council of Norway (Småforsk grant) and an NTNU Mobility Fellowship. 
[1] P. Tran, V. Celli, and A. A. Maradudin, J. Opt. Soc. Am. A11, 1686 (1994).

[2] R. F. Harrington, Field Computation by Moment Methods (Macmillan, New York, 1968).

[3] R. L. Wagner, J. Song, and W. C. Chew, IEEE Trans. Antennas Propag. 45, 235 (1997).

[4] L. Tsang, C. H. Chan, and K. Pak, Electron. Lett. 29, 1153 (1993).

[5] L. Tsang, C. H. Chan, and K. Pak, J. Opt. Soc. A11, 711 (1994).

[6] K. Pak, L. Tsang, C. H. Chan, and J. T. Johnson, J. Opt. Soc. Am. A12, 2491 (1995).

[7] J. T. Johnson, L. Tsang, R. T. Shin, K. Pak, C. H. Chan, A. Ishimaru, and Y. Kuga, IEEE Trans. Antennas Propag. 44, 748 (1996).

[8] D. Torrungrueng, H. -T. Chou, and J. T. Johnson, IEEE Trans. Geosci. Remote Sensing 38, 1656 (2000).

[9] G. Soriano and M. Saillard, J. Opt. Soc. Am. A18, 124 (2001).

[10] H. van der Vorst, SIAM J. Sci. Statist. Comput. 13631 (1992).

[11] A. A. Maradudin, T. Michel, A. R. McGurn, and E. R. Méndez, Ann. Phys. (N.Y.) 203, 255 (1990), Appendix A.

[12] J. A. Kong, Electromagnetic Wave Theory, 3rd ed. (EMW Publishing, Cambridge, MA, 2005), pp. 674-675.

[13] W. H. Press, S. A. Teukolsky, W. T. Vetterling, and B. P. Flannery, Numerical Recipes in Fortran, second ed. (Cambridge University Press, New York, 1992), p. 129.
[14] Y. Saad and M.H. Schultz, SIAM J. Sci. Stat. Comput. 7, 856 (1986).

[15] J.E. Sipe, J. Opt. Soc. Am. B, 4, 481 (1987).

[16] A.R. McGurn, A.A. Maradudin and V. Celli, Phys. Rev. B 31, 4866 (1985).

[17] E.R. Méndez and K.A. O’Donnell, Opt. Commun. 61, 91 (1987).

[18] D. Torrungrueng and J.T. Johnson, J. Opt. Soc. Am. A 18, 2518 (2001).

[19] E.I. Chaikina, P. Negrete-Regagnon, V. Ruiz-Cortés, E.R. Méndez, Opt. Commun. 208, 215 (2002).

[20] When in the text discussing the results of Figs. 2 and 3 we follow the sign convention for $\theta_{s}$ introduced in the caption of Fig. 2. Elsewhere, however, the standard spherical coordinate convention $\left(\theta_{s} \geq 0^{\circ}\right)$ will be followed.

[21] Note that we from now and onwards will adapt standard spherical coordinates so that $\theta_{s} \geq 0^{\circ}$.

[22] The simulation results reported herein assumed an azimuthal angle of $\phi_{0}=0^{\circ}$ which also determines the directions of the electric field vector associated with the incident Gaussian beam, and also defines (in our convention) the rotation angle of the incident plane. Another choice for $\phi_{0}$ would consequently also alter the orientation of the dipole-like patterns.

[23] This time is lower than that reported in Table II since only the scattered field in either the in-plane or out-ofplane configuration was calculated. 